Statistica Neerlandica (2003) Vol. 57, nr. 4, pp. 484-508

\title{
An Empirical Study of Cash Payments
}

\author{
Jeanine Kippers \\ Currency Policy Department, De Nederlandsche Bank
}

Erjen van Nierop

Graduate School of Industrial Administration, Carnegie Mellon University

Richard Paap

Econometric Institute, Erasmus University Rotterdam

Philip Hans Franses*

Econometric Institute, Erasmus University Rotterdam

Anytime an individual makes a cash payment, he or she needs to think about the amount to be paid, the coins and notes which are available, and the amount of change. For central banks and retail stores, it is of interest to understand how this individual choice process works. The literature of currency use concerns primarily theory, in the sense that, given certain assumptions, one can derive an optimal denomination range. There is no empirical study which deals with the actual use of coins and notes, given a specific denomination range.

In this paper we present such a study, which is based on two rather unique data sets. We use descriptive statistics and a sophisticated model, which is designed for this specific purpose, to see whether two basic premises of the theories on optimal ranges are valid. In contrast to the widely accepted assumptions, we find that individuals appear not to pay efficiently and that they are also not indifferent to the use of coins and notes. In other words, some notes and coins are used less often than expected given the payment situation.

Key Words and Phrases: Cash payment, efficiency, denomination range.

"Some people still have to get used to the Euro. A gas station cashier in Erbach, Germany, turned out not to be completely familiar with the new denominations. A client paid with a note of 300 Euro, and the cashier happily returned him the amount of 250 Euros."

NOS Teletext Page 192, May 312002

\footnotetext{
*We thank participants of the Joint Statistical Meeting 2001 in Atlanta for their helpful comments.
} 


\section{Introduction}

A case of Euro forgery occurred five months after its introduction in Germany, as the above news flash indicates. Interestingly, the fake Euro note does not have the face value of 200 or 500 , but 300 . Hence, anyone familiar with the Euro notes could have recognized that the note must be fake. But perhaps the number 300 is not chosen at random by the forger. Currency theory suggests that the optimal range of denominations is either based on factors of 2 or 3 . So, the forger must have thought that the 300 Euro note would perhaps not look that strange in a cash payment, even though a 400 Euro note would have resulted in more profit. In other words, some denominations are perhaps treated differently in cash payments, and it is this issue that we study in the present paper.

Many individuals make cash payments each day. Even though plastic money has gained a substantial market share, there are many everyday situations where we need coins and notes to make a payment. The values of these coins and notes are determined from the outset, which are usually values such as 1, 2, 5 and 10 and multiples thereof, but the contents of our wallets tend to be rather random. Hence, any time we need to make a payment, we need to think about the amount to be paid, the coins and notes we have, and the value of these which we will get in return if we cannot exactly match the transaction amount. In other words, the decision of how we decide to use coins and notes amounts to an individual choice process.

Of course, central banks and retail stores are just two examples of those who have (or should have) an interest in the cash payment process. Central banks issue notes and coins, and the production and distribution process is not cheap. Notes or coins that are issued but rarely used in practice could turn out to be an expensive choice made by central banks. On the other hand, those denominations that are used more often than expected might cause less efficient payments and result in a more expensive currency system than was anticipated by the central bank. This concerns not only the local banks that provide individuals with cash, but also retail stores which need to provide the cashiers with the required cash for the daily transactions.

The main approach to handling these issues for, predominantly, central banks is governed by the idea that the range of coins and notes should obey criteria concerning efficiency and the amount of computational efforts required by the paying individual. The importance of this matter is recognized in currency research and it receives its rightful attention in the relevant literature. The trade-off which is faced by central banks is transformed into diverse optimization problems leading to a variety of ideas on what is called the optimal denomination structure. The solution to this optimizing problem, which is of particular importance to central banks is not beyond discussion, see for example Hove (2001).

There are two views on the optimizing criterion applicable to this problem. First, one can assume an analogy with the problem of Bachet, which concerns the optimal set of standard weights. According to this approach, the optimal range of denominations of notes and coins has the smallest number of denominations with 
which one can pay any amount in between the smallest transaction and a certain upper bound, see Telser (1995) and WynNe (1997) for a discussion of this approach. Assuming that all denominations have the same cost of production and that payment amounts follow a uniform distribution, this range of denomination will be the cheapest for the issuing authority.

The second view, which is recently summarized in Hove (2001), adopts the "principle of least effort". That is, the optimal range of denominations allows the number of tokens exchanged in cash transactions to be minimized, on average, see Caianiello et al. (1982), Sumner (1993) and Hove and Heyndels (1996), among others. The two different views on how to derive the optimal currency system has led to the result that denominations should be spaced apart by a factor of either two or three, but no firm conclusion has been reached.

The literature on currency research is predominantly of a theoretical nature. Interestingly, at least as far as we know, there are no empirical studies on cash payments. That is, there are no studies which try to answer questions like: "Do some coins get used more often than others?", or "How do individuals choose between the coins and notes in their wallets?" Of course, one can just simply count the number of times certain coins and notes are distributed and, after a while, returned to the central bank. However, this assumes that all notes and coins are equally relevant for each possible transaction. Also, it fails to assume that people collect notes and coins, that money can get lost, and that the wallets of individuals do not always contain the relevant denominations for all possible payments. In sum, it would be of interest to see what people actually do when they make cash payments. This investigation should then go beyond mere descriptive statistics, and should follow a modeling approach taking the mentioned issues into account. It is the purpose of our paper to perform such an investigation, which, to our knowledge, is the first of its kind.

Our paper centers around two data sets that enable us to perform the empirical investigation. The first data set concerns a large number of actual transactions where we know: the amount that an individual has to pay, how he or she paid, and what they got in return. Unfortunately, due to privacy reasons, we have no information on the actual content of the wallets prior to the payment. This motivates the collection of a second data set, which is based on an e-mail survey, to get an impression of the typical contents of wallets. The data we use for our empirical analysis are described in Section 2 of this paper.

We use descriptive techniques and statistical modeling in our analysis of these two data sets. The prime focus of our analysis concerns the basic premises on individual payment behavior of the theories discussed above. The two basic premises are: (i) that individuals use notes and coins in an efficient way, and (ii) that individuals are indifferent towards to use of specific coins and notes. These premises can be put under scrutiny if one has actual data, as we have. For theoretical arguments, for which we appreciate the relevance, the theories furthermore assume: (iii) that individuals have access to all the relevant possible combinations, and (iv) that all payment amounts are equally likely to occur. The nature of our analysis of premises (c) VVS, 2003 
(i) and (ii), using actual data on payments and wallets, allows us to take a more realistic approach to cash payment analysis. That is, actual payment amounts are studied, and restricted use of notes and coins is accounted for with the use of data on wallet contents.

With the cash payment data set, we examine in Section 3 if individuals have a tendency to pay efficiently. Next, we examine premise (ii), and the relevant discussion appears in Section 4. For this purpose we develop a specific statistical model, for which in Section 5 we discuss its representation, its interpretation and parameter estimation. The specific form of the model is established by the fact that a payment process involves huge numbers of possible choices, and also by a property of our data, which is that we do not observe the actual wallets at the time of payment. In Section 6, we consider our model for the data, and we show how its interpretation suggests that, for the specific data, certain notes and coins are chosen too infrequently, given other coins and notes and given the transaction properties. Our empirical analysis suggests a wide range of further research topics, which we outline in the concluding section of this paper.

\section{Data}

For this study we make use of two main data sets. The first concerns cash transactions. It contains a random sample of a large number of cash payments registered on the authority of the Nederlandsche Bank in 1998. This survey was intended to gain insight into the number of banknotes and coins that are used daily in over-the-counter cash payments, for a thorough preparation of the Euro changeover in the Netherlands. For this specific purpose two methods of data collection were considered. Minimal effort and cost would be involved in having a representative group of citizens keep a diary of their cash payments over, say, a week. These individuals are asked to register the amount to be paid and, the different banknotes and coins used, as well as change for all cash payments. A representative sample of transactions is most easily attained by selecting a representative group of individuals. For the second method, observers were stationed at points-of-sale to register each cash payment at the counter. For reasons of precision the second method of data collection was chosen. It was feared that in the first method the resulting data set would be biased towards cash payments with higher amounts as individuals could be expected to be more oblivious to amounts under, say, NLG 10 (equivalent to $€ 4.54$ ). As these amounts are equally relevant to the research, the first method was considered less favorable.

Retail outlets were selected in municipal and rural areas spread over the country. Different types of retail outlets were included, ranging from supermarkets and warehouses to bakeries and shoe stores. Cash payments were registered over a week to account for weekly and daily fluctuations. Furthermore, the number of cash payments resulting from a week of surveying was expected to be sufficient to make a (C) VVS, 2003 
judgement on the use of notes and coins in cash payments for each of four retail categories, that is, supermarkets, department stores, specialized food stores and nonfood stores. Special permission was given by the management of 69 retail outlets to observe payments at one of their cash registers. The retail outlets were anonimized in such a way that only the retail category was registered. For privacy reasons no additional data other than the payment characteristics were collected for the paying individuals. The survey resulted in a data set of 40,610 payments, of which 38,330 were cash payments. We randomly selected 2,000 cash payments from the data set, from which we excluded the so-called added payments. These are payments in which small change is added to the payment to simplify the change returned by the cashier. This behavior does not constitute the central issue of this paper, and therefore these added payments are not included in the analysis. The 2,000 cash payments constitute the main data set of our study. For each payment the data set contains the payment amount, the notes and coins that were used to pay the amount, and the notes and coins received as change in the case of overpayment. In the Netherlands, banknotes in 1998 were issued in six denominations, that is, $1000,250,100,50,25$ or 10 guilders. Coins were issued in six denominations, that is, 5, 2.5, 1, 0.25, 0.10 and 0.05 guilders.

For the analysis presented in this paper, we also collected additional data on individuals' wallets, to account for the unknown wallet content at the time of the transactions in our cash payment data set. Data were collected through an e-mail survey. Out of 1500 surveys, 840 were returned. The respondents were asked to register the number of banknotes and coins in their wallet at the time of registration. The highest banknote denominations were excluded from the survey to attain a higher response. As these are held as in reserve rather than for transaction purposes, respondents could feel their privacy to be invaded, while data on these denominations were not considered to be of importance to the study. Analysis of the wallet contents, in connection to individual characteristics such as age and gender, showed no strong sign of underrepresentativeness. The survey resulted in a data set of 840 wallets, in the following referred to as the wallet contents data set.

\section{Efficiency in cash payments}

Our cash payment data set has actual transactions, and it is tempting to put a basic premise under scrutiny that has been widely used in theoretical currency research. Various articles, as referred to in the introduction, are concerned with deriving the optimal denominational structure as a solution to a theoretical optimizing problem in which the optimizing criterion is efficiency. According to this view, the optimal currency system would allow efficient payments with the smallest number of banknotes and coins, on average. For solving this problem it is needed to assume that individuals behave accordingly. In other words, individuals, when facing cash payments, are assumed to make the necessary computations to pay an amount with (c) VVS, 2003 
the smallest number of banknotes and coins. In addition, for theoretical arguments, it is implicitly assumed that individuals would have access to all the relevant possible combinations.

These premises, referred to earlier as premise (i) and (iii) in currency research in section 1, make sense from a theoretical point of view. Premise (i) concurs with rational behavior and it can be assumed that individuals would at least strive for some degree of efficiency. Premise (iii) however is obviously false in practice. It is however necessary to stick to (iii) to derive a solution to the optimization problem. In this section we intend to explore payment behavior as regards to efficiency, while accounting for a limited access to notes and coins, making use of our main data sets with actual cash payments and wallet contents.

As a starting point we are guided by theoretical concepts introduced by CRAMER (1983). In order to assess the merits of the Dutch currency system, Cramer presents a method of relating the actual demand for banknotes and coins at a macroeconomic level to their average use in cash transactions. For example, applying his method to the Dutch currency system in 1982, he finds that the higher denominations circulate in larger numbers than would be expected on the basis of their average use in cash transactions. This indicates the use of banknotes for other than transaction purposes. For calculating average use in cash transactions, Cramer develops a theoretical model for payment behavior applying the so-called principle of least effort to individual transactions. He defines an 'efficient payment' to be the payment of an arbitrary amount in which the number of banknotes and coins exchanged are minimized.

Any amount has one or more payment schemes, as can be seen from the following example. The amount NLG 17.50 has three efficient payment schemes in which three tokens are used. Any other combination will need more than three tokens to make up the amount NLG 17.50.

- Payment 1: NLG $10+$ NLG 5 + NLG 2.50

- Payment 2: $2 \times$ NLG 10 and NLG 2.50 as change

- Payment 3: NLG 25 and NLG $5+$ NLG 2.50 as change

Cramer develops an algorithm for determining all efficient payment schemes for a range of payment amounts and discovered that no Dutch amount up to NLG 100.00 would need more than seven tokens to make the payment and that the amount NLG 16.65 had the most efficient schemes, that is, ten in total.

We aim to draw conclusions on efficient payment behavior by comparing the number of notes and coins used in actual payments to the efficient number. As Cramer did, we define efficient payments as all payment schemes in which the number of notes and coins that form a payment amount, including change, is minimized, but add the restriction that the paying individual only has access to the content of his or her own wallet. This concept is somewhat different from the one in CRAMER (1983) in the sense that restricted efficient payment schemes vary across wallets. This means that, for each wallet, different efficient payment schemes hold. (c) VVS, 2003 
Table 1. Frequency distribution of inefficiencies defined as the number of notes and coins used more than is needed for wallet-specific efficient payment.

\begin{tabular}{|c|c|c|c|}
\hline Range & $\begin{array}{l}\text { Number of } \\
\text { individuals }\end{array}$ & $\begin{array}{l}\text { Cumulative } \\
\text { number of } \\
\text { individuals }\end{array}$ & $p$-value ${ }^{\mathrm{a}}$ \\
\hline 0.0 & 73 & 73 & 1.00 \\
\hline $0.0-0.5$ & 16 & 89 & 0.84 \\
\hline $0.5-1.0$ & 41 & 130 & 0.00 \\
\hline $1.0-1.5$ & 12 & 142 & 0.00 \\
\hline $1.5-2.0$ & 15 & 157 & 0.00 \\
\hline $2.0-2.5$ & 7 & 164 & 0.00 \\
\hline $2.5-3.0$ & 11 & 175 & 0.00 \\
\hline$>3.0$ & 18 & 193 & 0.00 \\
\hline \multicolumn{4}{|c|}{$\begin{array}{l}\text { a To check whether the cumulative number of } \\
\text { individuals constitutes a significant fraction of the } \\
\text { sample of } 193 \text { individuals, we use the binomial sign test. } \\
\text { Under the null-hypothesis } H_{0} \text {, the probability of paying } \\
\text { efficiently is the same as for paying inefficiently, that } \\
\text { is Pr[efficient] }=\operatorname{Pr}[\text { inefficient] }=0.5 \text {. The alternative } \\
\text { hypothesis } H_{1} \text { states that the probability of paying } \\
\text { efficiently is higher than the probability of paying } \\
\text { inefficiently. }\end{array}$} \\
\hline
\end{tabular}

For this reason, we adjust the algorithm in CRAMER (1983) to compute the efficient payment scheme to meet the extra restriction of the wallet. This is described in detail in the appendix.

For each payment in a sample of amounts from our cash payment data set, we compute wallet-specific efficient payment schemes with randomly selected wallets from the wallet contents data set that are feasible for the payment. Due to the complexity and time-consuming character of the algorithm, we limit ourselves to 193 cash transactions from the 2,000 transactions in the cash payment data set. We generate efficient payment schemes for these transactions, for ten different wallets each. We compare the actual payments with the efficient payment schemes for each wallet and compute the difference in numbers of notes and coins used. Table 1 presents the frequency of differences averaged over 10 wallets.

The table shows that 73 individuals, nearly 40 percent of the sample, pay with no inefficiency. To check whether this percentage constitutes a significant fraction of the sample, we use the binomial sign test. Under the null hypothesis $H_{0}$ the probability of paying efficiently is the same as paying inefficiently, that is $\operatorname{Pr}[$ efficient] $=$ $\operatorname{Pr}[$ inefficient $]=0.5$. The alternative hypothesis $H_{1}$ states that the probability of paying efficiently is higher than the probability of paying inefficiently. Under $H_{0}$ the number of efficient payments has a binomial distribution with parameters $n=193$ (number of transactions) and $P=0.5$.

For the 73 efficient transactions out of 193 individuals mentioned above, the $p$-value of the sign test equals 1 . This tells us that this number does not constitute a significant proportion of the sample, and thus we cannot reject $H_{0}$. So, if we were to 
test whether individuals pay perfectly efficiently, the data tell us this is not the case, as this would have meant that the figure in the first row should have been closer to 193. When applying a less strict definition of efficiency, for example allowing an inefficiency of 0.5 tokens, the number increases to 89 individuals, which is still insignificant. The $p$-value of the sign test becomes smaller than 0.05 at an inefficiency of 0.7 , when 108 individuals pay with an inefficiency of 0.7 at maximum. This means that there is a significant number of individuals who use only 0.7 or less redundant denominations when making payments, taking into account the content of the wallet. This can be interpreted as a sign that individuals do strive for efficiency, indicating rational economic behavior, but other reasons exist for the choice of a less than efficient payment scheme in many circumstances.

\section{Towards an econometric model}

From the previous section it can be concluded that although efficiency explains payment behavior to some degree, it does not fully explain the choice of notes and coins in cash payments. It seems that influences other than efficiency account for the observed cash payments. Furthermore, so far we have not paid attention to differences in the use of banknotes and coins, apart from their most apparent characteristic. The face value is the only feature that determines the utility of a note or coin in efficient payment schemes. The question is now whether such an indifference towards denominations, apart from their nominal value, which was referred to as premise (ii) in the Introduction, is plausible? One would expect equal treatment of notes and coins in equal circumstances. At least, in composing an efficient denominational structure, this is assumed by a central bank. Such a bank aims to provide for the most efficient cash payment system. Given the database, we are now challenged to explore premise (ii) further. Specifically, we want to answer the following question: are some denominations more or less preferred than others?

From a macroeconomic point of view, the demand for currency is represented by its total number in circulation. From these figures - generally available to central banks on a day-to-day basis - it is clear which denominations have the highest overall demand. However, these figures do not represent the use of notes and coins in cash transactions. Given that we are studying the use of Dutch guilder notes and coins in 1998, we present in Table 2 the end-of-month circulation figures for the Dutch guilder in March 1998 to make our point.

The demand for the highest denominations is mainly determined by their role as a means of saving, which is estimated to be around 70 percent of the number in circulation. The NLG 50 note has a relatively low demand in comparison with its neighboring denominations NLG 100 and NLG 25. Is this note somewhat unpopular? The high valued note NLG 100 is used as a means of payment as well as a means of saving, and some of the NLG 25 might have been lost over the years, causing the demand figure to be distorted. Such loss is a rather common (C) VVS, 2003 
Table 2. Circulation figures for Dutch guilders as at the end of March 1998, in millions of pieces.

\begin{tabular}{lclll}
\hline Notes & & & Coins \\
\cline { 1 - 2 } \cline { 5 - 5 } Denomination & In circulation & & Denomination & In circulation \\
\hline 1000 & 14 & 5 & 190 \\
250 & 20 & & 2.5 & 188 \\
100 & 138 & & 688 \\
50 & 33 & 0.25 & 1260 \\
25 & 76 & 0.10 & 2122 \\
10 & 82 & 0.05 & 1560
\end{tabular}

Table 3. Average number of notes and coins used in payments, calculated from the cash payment data set containing the sample of 2,000 retail payments.

\begin{tabular}{lllll}
\hline Notes & & & Coins \\
\cline { 1 - 1 } Denomination & Average number & & Denomination & Average number \\
\hline 1000 & 0.001 & 5 & 0.181 \\
250 & 0.007 & 2.5 & 0.089 \\
100 & 0.137 & 1 & 0.431 \\
50 & 0.099 & 0.25 & 0.290 \\
25 & 0.283 & 0.10 & 0.248 \\
10 & 0.333 & 0.05 & 0.160 \\
\hline
\end{tabular}

phenomenon in particular for coins, given their low value and long circulation since 1945. With these effects, along with the possibility of a high number of coins kept in savings, it should be evident that no solid conclusion can be drawn on the use of coins and notes in cash payments on the basis of circulation figures. It is our strong belief that in order to examine premise (ii), one needs to focus on individual payments. As we have actual data on cash payments, we explore methods of making comparisons between denominations using the data at hand.

As it turns out, the question whether all denominations are treated equally by individuals in cash payments is not easily answered, not even if you have actual data, as we do. If the number of times denominations are used in cash payments is simply counted, the resulting frequency of use does not lead to any meaningful conclusions. We illustrate this by regarding the frequency of use for the Dutch guilder, denominations calculated from our cash payment data set, containing 2,000 payments, as presented in Table 3 below.

From the figures in Table 3 it is obvious that the NLG 1000 and NLG 250 banknotes are rarely used in cash payments. As the average payment amount in the sample was NLG 18, this is not a surprising result. Furthermore, an unreported histogram of the payment amounts shows that the distribution is not uniform. This finding rejects premise (iv) mentioned in section 1. It is also apparent that three denominations can be distinguished as having a low frequency of use as compared with their neighboring denominations. First, the NLG 50 has a lower frequency than 
both NLG 100 and NLG 25. This is in line with our observation from the circulation figures at a macroeconomic level. Second, in comparison with the banknote with the lowest denomination, the highest coin NLG 5 shows a low frequency, while the use of the NLG 2.50 is even lower. In all, one could conclude that three denominations seem to be less preferred from these descriptive statistics.

However, such firm conclusions cannot be drawn on the basis of these simple statistics. Different situations lead to different uses of denominations. For example, the use of a NLG 100 note is lower for a payment amount of NLG 5.00 than for NLG 90.00. Next, if the NLG 5 is better represented in the wallets of individuals than NLG 2.50, it cannot be concluded that NLG 2.50 is less popular in cash payments. The individual simply has less opportunity to choose the NLG 2.50. When such specific circumstances at the time of payment need to be taken into account for truly judging the use of denominations, one needs to conclude that this can only be done by means of statistical modeling. The next section will focus on the development and estimation of such a model consistent with the specific characteristics of cash payments, while thereafter it is shown that the estimation results give us the answer to the question of which denominations in the Dutch range were more or less preferred in 1998.

\section{The model}

In this section we outline our model for currency use, which takes the payment amount and wallet contents into account.

\subsection{Preliminaries}

The focus of this study is on individual cash payments from the viewpoint of a payer $i$. In this sense the payer faces a choice problem. A certain amount $A_{i}$ has to be paid and the paying individual disposes of a set of banknotes and coins in his or her wallet. Which of these banknotes and/or coins will he or she select to fulfill the payment? We aim to model this choice.

The choice is described by the $D$-dimensional discrete random variable $Y_{i}$, where $D$ corresponds to the number of denominations in a range. This random variable describes the number and combination of coins and banknotes selected by the individual $i$ to pay amount $A_{i}$. The realizations of this random variable are denoted by $y_{i}$. Our goal is to describe the probability process for $Y_{i}$.

The possible values that the random variable $Y_{i}$ can take depend of course on the content of the wallet of individual $i$. Let $W_{i}$ be a similar $D$-dimensional random variable describing the contents of the wallet of payer $i$. That is, $W_{i}$ represents the number of notes and coins that are available to the paying individual at the moment of payment. Suppose for now that the value of the random variable $W_{i}$ is known and denoted by $w_{i}$. We aim to model the probability of outcome $y_{i}$ if amount $A_{i}$ has to be paid, given the content of the wallet $w_{i}$, that is, 


$$
\operatorname{Pr}\left[Y_{i}=y_{i} \mid w_{i}\right]
$$

The probability model describing $Y_{i}$ has to fulfill certain requirements. For example, it is not possible that a payer $i$ pays with denominations which are not in his wallet. Hence, this imposes the restriction on $Y_{i}$ that $Y_{d, i}$ is smaller than or equal to $w_{d, i}$ for each denomination $d=1, \ldots, D$. Furthermore, we have to impose the condition that the monetary value of $Y_{i}$ has to be larger than or equal to $A_{i}$. The conditions for an appropriate probability model can be summarized as follows:

(a) The value of $Y_{d, i} \leqslant w_{d, i}$ for $d=1, \ldots, D$.

(b) The probability of choosing $y_{i}$ when the monetary value of $y_{i}$ is lower than $A_{i}$ is zero.

(c) The probability of choosing $y_{i}$ when elements of $y_{i}$ are expected to be returned as change is zero.

(d) If there is only one possibility of paying amount $A_{i}$ given $w_{i}$, then the probability of choosing this $y_{i}$ is 1 .

(e) The probabilities of all feasible values of $Y_{i}$ given $w_{i}$ sum up to 1 .

We use an example for illustration. Assume a virtual denominational range consisting of banknotes with nominal values $(100,25,10,5)$. Furthermore, assume that the paying individual has one banknote each of 25,10 and 5 in his or her wallet, that is, $w_{i}=(0,1,1,1)$, and that an amount of 15 has to be paid. In this situation, the paying individual has the following options that are consistent with conditions (a) and (b), that is, the wallet contains the relevant banknotes and the value is higher than or equal to 15 .

- $(0,1,0,0)$ with value 25

- $(0,1,1,0)$ with value 35

- $(0,1,1,1)$ with value 40

- $(0,0,1,1)$ with value 15

From this list it is obvious that the individual will not choose $(0,1,1,0)$ or $(0,1$, $1,1)$ as these options include 'unnecessary' notes, consistent with condition (c). That is, when paying with $(0,1,1,0)$ for amount 15 , the note of value 10 will also be returned as change. A payment of $(0,1,0,0)$ will suffice. In this example the only remaining payment options are $(0,1,0,0)$ and $(0,0,1,1)$. The paying individual will choose either one or the other, consistent with condition (e). If the wallet had not contained a banknote of 25 , that is $w_{i}=(0,0,1,1)$, then only one payment option would remain, that is, $(0,0,1,1)$. The payer would have no choice but to use this combination, as stated by condition (d).

Given these conditions for an appropriate probability model for cash payments, we have to conclude that standard discrete choice models do not apply. As a denominational range usually consists of 12 or more denominations, possible outcomes of $Y_{i}$ can easily total up to a large number. Also, each payment amount $A_{i}$ 


$\operatorname{Pr}\left[Y_{100}=y_{100}\right]$
$\operatorname{Pr}\left[Y_{25}=y_{25}\right.$ । $\left.y_{100}\right]$
$\operatorname{Pr}\left[Y_{10}=y_{10} \mid y_{100}, y_{25}\right]$
$\operatorname{Pr}\left[Y_{5}=y_{5} \mid y_{100}, y_{25}, y_{10}\right]$
possible outcomes

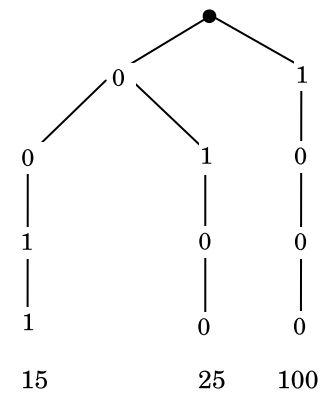

Fig. 1. Nesting structure of our model.

and each wallet $w_{i}$ generates a different choice set for the payment choice. It would therefore be inconceivable to assign a probability to each possible outcome of $Y_{i}$. To by-pass this, we suggest decomposing $\operatorname{Pr}\left[Y_{i}=y_{i} \mid w_{i}\right]$ into a sequence of conditional probabilities describing the probability of the individual elements of $Y_{i}$. For each of the conditional probabilities we can formulate an appropriate probability model. We use the following decomposition to allow for the interdependency of the different elements of $Y_{i}$

$$
\begin{aligned}
& \operatorname{Pr}\left[Y_{i}=y_{i} \mid w_{i}\right]=\operatorname{Pr}\left[Y_{D, i}=y_{D, i} \mid w_{i}\right] \operatorname{Pr}\left[Y_{D-1, i}=y_{D-1, i} \mid y_{D, i}, w_{i}\right] \\
& \operatorname{Pr}\left[Y_{D-2, i}=y_{D-2, i} \mid y_{D, i}, y_{D-1, i}, w_{i}, \cdots \operatorname{Pr}\left[Y_{1, i}=y_{1, i} \mid y_{D, i}, \ldots, y_{2, i}, w_{i}\right] .\right.
\end{aligned}
$$

Hence, we first model $Y_{D, i}$ given $w_{i}$. Next, we model $Y_{D-1, i}$ given $w_{i}$ and $y_{D, i}$, and so forth.

Using this decomposition, it is easier to incorporate the restrictions on the $Y_{i}$ variable and on the probabilities. This is illustrated in Figure 1, again considering the example mentioned above with a virtual denominational range consisting of four banknotes with nominal values 100, 25, 10 and 5 and an amount to be paid of 15 . Now, we assume that the paying individual has one banknote of each in his or her wallet, that is, $w_{i}=(1,1,1,1)$. The nesting structure as depicted in Figure 1 would satisfy the above mentioned conditions where three possible outcomes result.

If the banknote of 100 is already chosen by the individual, the probability of choosing 25, 10 and 5 is zero as 100 already exceeds the payment amount 15. If neither banknote 100 nor 25 has been chosen, the probability of choosing both 10 and 5 is 1 as this is the only remaining option to form the payment amount.

\subsection{The statistical model}

The next step of our modeling strategy concerns the specification of the individual probabilities in (2). Let us first consider modeling $Y_{D, i} \mid w_{i}$. The maximum number of notes of type $D$ that can be used for paying $A_{i}$ is $w_{D, i}$, consistent with condition (a) for an appropriate payment model. However, as condition (c) states, banknotes and coins will not be chosen if they are expected to be returned as change. Thus, if the 
nominal value of the $d$-th denomination is denoted by $v_{d}$, the number of notes of type $D$ an individual can use for paying $A_{i}$ is bounded from above by

$$
u b_{D, i}=\min \left(\operatorname{ceil}\left(\frac{A_{i}}{v_{D}}\right), w_{D, i}\right) .
$$

Although the natural lower bound for $Y_{D, i}$ would be zero, it might be needed to use at least one note of type $D$ to be able to meet condition (b). For example, consider the situation where an individual has to pay an amount of NLG 130.00, and his/her wallet contains three notes, two with a nominal value of 100 and the other with a nominal value of 50 . Then the individual has no choice but to pay at least with one note of 100 to be able to make the payment. In general, an individual $i$ has to pay with $k$ denominations $D$ if the difference between $A_{i}$ and the monetary value of the denominations $D-1$ to 1 is more than $k$ times the monetary value of note $D$. Hence, the choice range of individual $i$ is bounded from below by

$$
l b_{D, i}=\max \left(\operatorname{ceil}\left(\frac{A_{i}-\operatorname{amount}\left(w_{1, i}, \ldots, w_{D-1, i}\right)}{v_{D}}\right), 0\right),
$$

where the function amount $\left(x_{p}, \ldots, x_{q}\right)$ is defined as the monetary value of the denominations $x_{p}$ through $x_{q}$, that is,

$$
\operatorname{amount}\left(x_{p}, \ldots, x_{q}\right)=\sum_{k=p}^{q} v_{k} x_{k} .
$$

For denominations $D-1$ through 1 choice ranges apply in a similar manner. To construct the upper bound and lower bounds for denomination $d$, we have to correct for the monetary values of $y_{D, i}$ to $y_{d+1, i}$ as we condition on these values. The upper and lower bounds are given by

$$
l b_{d, i}=\max \left(\operatorname{ceil}\left(\frac{A_{i}-\operatorname{amount}\left(y_{d+1, i}, \ldots, y_{D, i}\right)-\operatorname{amount}\left(w_{1, i}, \ldots, w_{d-1, i}\right)}{v_{d}}\right), 0\right)
$$

and

$$
u b_{d, i}=\min \left(\operatorname{ceil}\left(\frac{A_{i}-\operatorname{amount}\left(y_{d+1, i}, \ldots, y_{D, i}\right)}{v_{d}}\right), w_{d, i}\right)
$$

for $d=D-1, \ldots, 1$.

Given the values of the upper bounds and lower bounds on each element of $Y_{i}$ we have to specify probabilities for the choice options of the individuals. We can distinguish two cases. If $l b_{d, i}=u b_{d, i}$, then the individual has no freedom of choice and hence

$$
\operatorname{Pr}\left[Y_{d, i}=y_{d, i}=l b_{d, i}=u b_{d, i} \mid y_{d+1, i}, \ldots, y_{D, i}, w_{i}\right]=1 .
$$

If $l b_{d, i}<u b_{d, i}$, we assume that $Y_{d, i}$ given $y_{d+1, i}, \ldots, y_{D, i}$ and $w_{i}$ is a truncated Poisson distributed on the region $\left[l b_{d, i}, u b_{d, i}\right]$ denoted by 


$$
Y_{d, i} \mid y_{d+1, i}, \ldots, y_{D, i}, w_{i} \sim \operatorname{POI}\left(\exp \left(x_{d, i}^{\prime} \beta_{d}\right)\right) \times I\left[l b_{d, i}, u b_{d, i}\right]
$$

where $\beta_{d}$ is a parameter vector and $x_{d, i}$ contains explanatory variables for denomination $d$. Hence, values of $Y_{d, i}$ outside the permitted region $\left[l b_{d, i}, u b_{d, i}\right]$ correspond to zero probability as they violate one or more of the conditions (a)-(e). In all, the conditional probabilities are defined as

$$
\operatorname{Pr}\left[Y_{d, i}=y_{d, i} \mid y_{D, i}, \ldots, y_{d+1, i}, w_{i}\right]=\left\{\begin{array}{cc}
\frac{\exp \left(-\exp \left(x_{d, i}^{\prime} \beta_{d}\right)\right) \exp \left(x_{d, i}^{\prime} \beta_{d}\right)^{y_{d, i}}}{c_{d, i}{ }^{\prime} y_{d, i} !} & \text { if } l b_{d, i} \leqslant y_{i, d} \leqslant u b_{d, i} \\
0 & \text { otherwise }
\end{array}\right.
$$

for $d=1, \ldots, D$, where $c_{d, i}$ corrects for the truncation and is given by

$$
c_{d, i}=\sum_{z=l b_{d, i}}^{u b_{d, i}} \frac{\exp \left(-\exp \left(x_{d, i}^{\prime} \beta_{d}\right)\right) \exp \left(x_{d, i}^{\prime} \beta_{d}\right)^{z}}{z !} \text {. }
$$

In the next section we discuss possible useful explanatory variables.

\subsection{Explanatory variables}

On the basis of the data available to us, we can specify a number of explanatory variables for (9). The main explanatory value is expected to be given by the payment amount. In our nested approach we define $A C O R R$ as the amount to be paid minus the value of the payments chosen for higher denominations, scaled to its face value. That is,

$$
\begin{aligned}
& \operatorname{ACORR}_{D, i}=\ln \left(\frac{A_{i}}{v_{D}}\right) \\
& \operatorname{ACORR}_{d, i}=\ln \left(\frac{A_{i}-\operatorname{amount}\left(y_{D, i}, \ldots, y_{d+1, i}\right)}{v_{d}}\right) \text { for } d=1, \ldots, D-1 .
\end{aligned}
$$

This variable can be seen as a scaled distance measure between the amount $A_{i}$ and the amount already paid with higher denominations. If the value of $A C C O R_{d, i}$ is high, this distance is large. The scaling with $v_{d}$ and the correction for the monetary value of the higher denominations allows us to compare the effect of the values of $A C O R R$ across denominations.

Furthermore, we define dummy variables that relate to the retail facility where the cash transactions took place. The transactions are observed in four identifiable retail branches. We define $D U M_{j, i}=1$ if the transaction of individual $i$ took place in facility $j$ and 0 otherwise.

\subsection{Unobserved wallet contents}

So far, we have modeled the cash payment probability conditional on the content of the wallet $w_{i}$. In our main data set, however, the wallet content of the individual at the time of the transaction is unknown. Therefore, we consider the wallet content as (C) VVS, 2003 
an unobserved random variable. The probability that the wallet of individual $i$ equals $w_{i}$ is denoted by $\operatorname{Pr}\left[W_{i}=w_{i}\right]$. The probability that individual $i$ chooses $y_{i}$ unconditional on the wallet content is the sum over all possible wallet contents multiplied by the probability that this wallet was the actual wallet, that is,

$$
\operatorname{Pr}\left[Y_{i}=y_{i}\right]=\sum_{w_{m} \in \mathcal{W}_{i}} \operatorname{Pr}\left[Y_{i}=y_{i} \mid w_{m}\right] \operatorname{Pr}\left[W_{i}=w_{m}\right],
$$

where $\mathcal{W}_{\mathrm{i}}$ denotes the set of feasible wallet contents for $y_{i}$.

It is difficult to specify a plausible probability model for the wallet contents. To obtain a plausible distribution we use a representative sample of feasible wallet contents consisting of $M$ observations, labeled as $r_{m}$ for $m=1, \ldots, M$. We use the empirical distribution of this a-select sample to model the distribution of $W_{i}$ in the following way

$$
\operatorname{Pr}\left[W_{i}=r_{m}\right]=\frac{1}{M} \text { for } m=1, \ldots, M .
$$

\subsection{Parameter estimation}

The likelihood function of our model equals the product of the choice probabilities over the $I$ individuals, that is,

$$
\begin{aligned}
L(y ; \beta)= & \prod_{i=1}^{I} \sum_{w_{m} \in \mathcal{W}_{i}} \operatorname{Pr}\left[Y_{D, i}=y_{D, i} \mid w_{m}\right] \operatorname{Pr}\left[Y_{D-1, i}=y_{D-1, i} \mid y_{D, i}, w_{m}\right] \\
& \ldots \operatorname{Pr}\left[Y_{1, i}=y_{1, i} \mid y_{D, i}, \ldots, y_{2, i}, w_{m}\right] \operatorname{Pr}\left[W_{i}=w_{m}\right],
\end{aligned}
$$

where $\beta=\left(\beta_{1}, \ldots, \beta_{D}\right)$ and $y=\left(y_{1}, \ldots, y_{I}\right)$. To estimate the model parameters we combine the likelihood function (15) with the wallet content probabilities (14) to the log likelihood function

$$
\begin{aligned}
l(y ; \beta) & =\sum_{i=1}^{I} \ln \left(\sum_{m=1}^{M} \operatorname{Pr}\left[Y_{D, i}=y_{D, i} \mid r_{m}\right] \ldots \operatorname{Pr}\left[Y_{1, i}=y_{1, i} \mid y_{D, i}, \ldots, y_{2, i}, r_{m}\right] \operatorname{Pr}\left[W_{i}=r_{m}\right]\right) \\
& =\sum_{i=1}^{I} \ln \left(\frac{1}{M} \sum_{m=1}^{M} \operatorname{Pr}\left[Y_{D, i}=y_{D, i} \mid r_{m}\right] \ldots \operatorname{Pr}\left[Y_{1, i}=y_{1, i} \mid y_{D, i}, \ldots, y_{2, i}, r_{m}\right]\right) .
\end{aligned}
$$

The parameters of this likelihood function can be estimated using standard optimization algorithms. For computational reasons, we average over a random sample of $K$ instead of over all $M$ wallets. Experiments with different values of $K$ showed that estimation results do not change much for values of $K \geqslant 10$. Under the usual regularity conditions, the ML estimator $\hat{\beta}$ is asymptotically normally distributed with the true value of $\beta$ as mean and covariance matrix equal to the inverse of the information matrix. To compute the covariance matrix we use the sandwich covariance estimator, which provides heteroskedastic-consistent standard errors, see WHITE (1980). 


\section{Estimation results}

We estimate the cash payment model, as specified by (2)-(9), for the cash payment data set. We exclude the highest denominations NLG 1000 and NLG 250 as data on wallet contents were collected without these denominations to avoid non-response. As these denominations are not primarily used as means of payment in retail transaction, they are also of less interest to our study. After removal of payments with these denominations a cash payment data set containing 1977 observations results for parameter estimation. For each observation, ten feasible wallet contents were randomly selected from the separate data set with wallet contents. We estimate three models, ranging from a basic model with only a constant, a second model including $A C O R R_{d}$ as explanatory variables and an elaborated model with dummy variables $D U M_{1}-D U M_{3}$ indicating the retail facility where the payment took place. Evaluating the three models by means of a likelihood ratio test, see Table 4, leads to the conclusion that the second model best represents the data as compared with the basic and elaborate model. It appears that the payment amount adds to the explanatory power of the model as compared with the basic Poisson model in which only a constant is included, while the subsequent inclusion of dummy variables does not. For further interpretation of our results we will use the second model as a reference. Its estimation results are presented in Table 5.

Table 4. Comparison of different model specifications.

\begin{tabular}{lllllll}
\hline Model & Explanatory variables & Loglik & Pseudo-R $^{2}$ & LR $^{\text {a }}$ & df & $p$-value \\
\hline 1 & Const & -36100 & - & - & \multicolumn{1}{c}{-} & - \\
2 & Const + ACORR & -35410 & 0.0191 & 1380 & 9 & 0.00 \\
3 & Const + ACORR + DUM & -35395 & 0.0195 & 30 & 27 & 0.31 \\
\hline
\end{tabular}

${ }^{\text {a }}$ The LR-statistic is computed to compare model 2 with model 1 , and model 3 with model 2.

Table 5. Parameter estimates with heteroskedastic-consistent standard errors in parentheses for the cash payment model.

\begin{tabular}{lcllll}
\hline & \multicolumn{2}{c}{ Constant } & & \multicolumn{2}{l}{ ACORR } \\
\cline { 6 - 6 } \cline { 5 - 6 } Denomination & Parameter & Standard error & & Parameter & Standard error \\
\hline 100 & 0.88 & $(0.06)$ & 1.56 & $(0.04)$ \\
50 & -0.59 & $(0.04)$ & 1.18 & $(0.03)$ \\
25 & 0.32 & $(0.03)$ & 1.41 & $(0.03)$ \\
10 & 0.05 & $(0.01)$ & 1.55 & $(0.04)$ \\
5 & 0.26 & $(0.04)$ & 1.78 & $(0.06)$ \\
2.5 & -1.27 & $(0.04)$ & 1.46 & $(0.06)$ \\
1 & 0.33 & $(0.03)$ & 0.66 & $(0.04)$ \\
0.25 & -0.71 & $(0.07)$ & 1.21 & $(0.09)$ \\
0.10 & 0.08 & $(0.02)$ & 1.90 & $(0.12)$ \\
\hline
\end{tabular}


It should first be noted that parameters for NLG 0.05 cannot be estimated due to our model specification, which imposes that the lower and upper bound are always equal for the lowest denomination. The parameter estimates of $A C O R R_{d}$ variables are significant and positive for all other denominations. A positive parameter estimate for $A C O R R_{d}$ would be expected, indicating that an increase in the payment amount will lead to an increase in the expected number of denominations $d$ chosen.

We use an example to illustrate the logic of this. Let us consider two situations. In the first an amount of NLG 155.00 has to be paid and the paying individual has already used a banknote of NLG 100 to pay for this amount. ACORR would be equal to 0.09 . In the second situation an amount of NLG 195.00 has to be paid, and again the paying individual has already used a banknote of NLG 100. ACORR would take on a higher value of 0.64 . The paying individual would have to pay a remaining amount of NLG 55.00 in the first case and a remaining amount of NLG 95.00 in the second case, with all the denominations lower than NLG 100 that are available to him or her. Given that the paying individual has the choice of paying with up to 2 banknotes of NLG 50, then the signs of the estimated parameters of $A C O R R$ indicate that the paying individual would be more inclined to pay with two banknotes of NLG 50 in the second situation than in the first.

To illustrate the effect of the $A C O R R_{d}$ variable on the expected value of $Y_{d}$, we plot in Figures 2-6 the expected value as a function of the $A C O R R_{d}$ variable for the different bank notes and coins. The lower bound is set equal to 0 , while the upper bound is set according to the value of the $A C O R R_{d}$ variable. Note that if we assume no wallet limitations, the upper bound is a function of the $\exp \left(A C C O R_{d}\right)$ variable

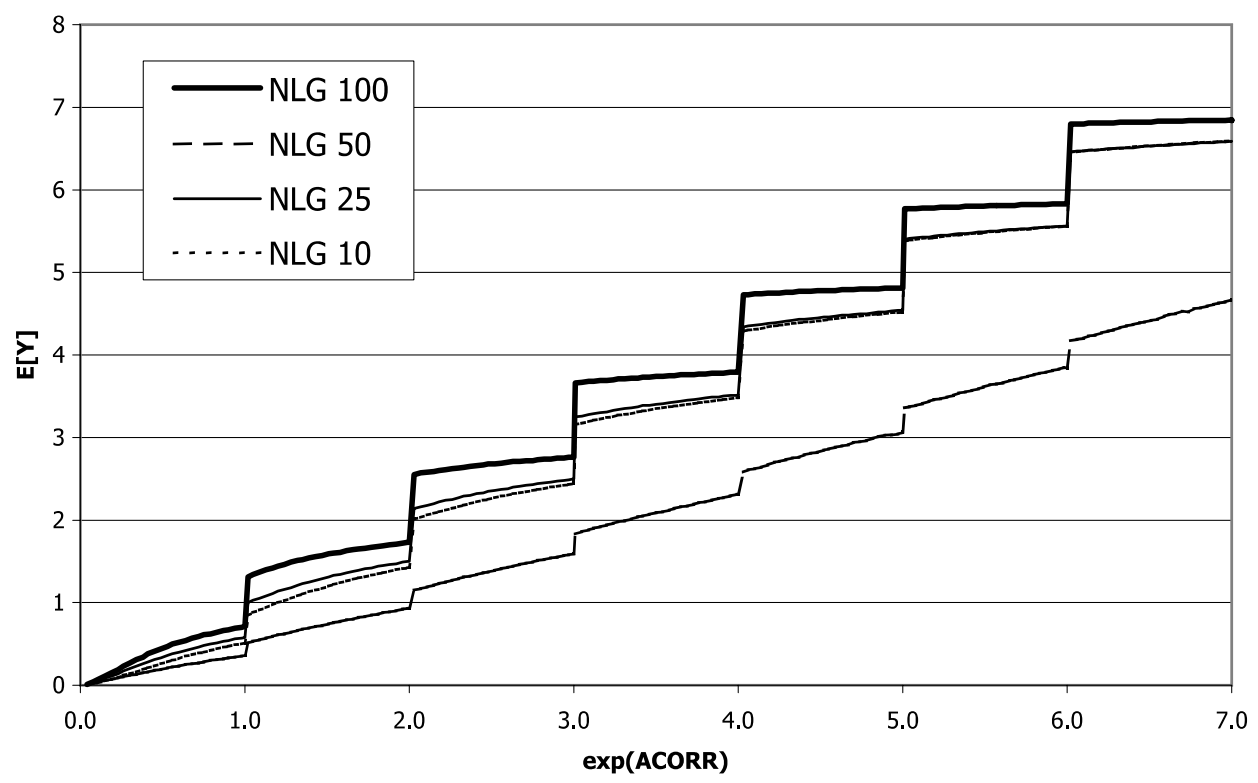

Fig. 2. Expected value of $Y_{d}$ for the notes (lower bound equals 0 ). 


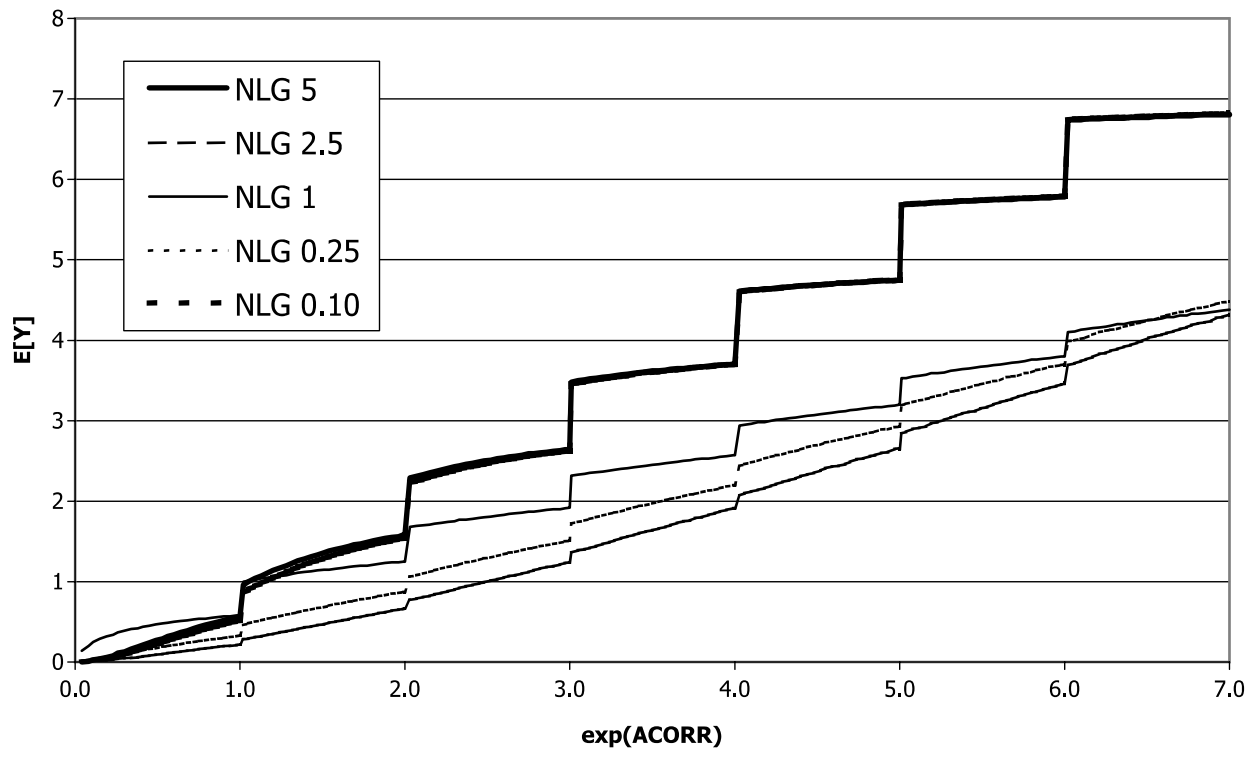

Fig. 3. Expected value of $Y_{d}$ for the coins (lower bound equal equals 0 ).
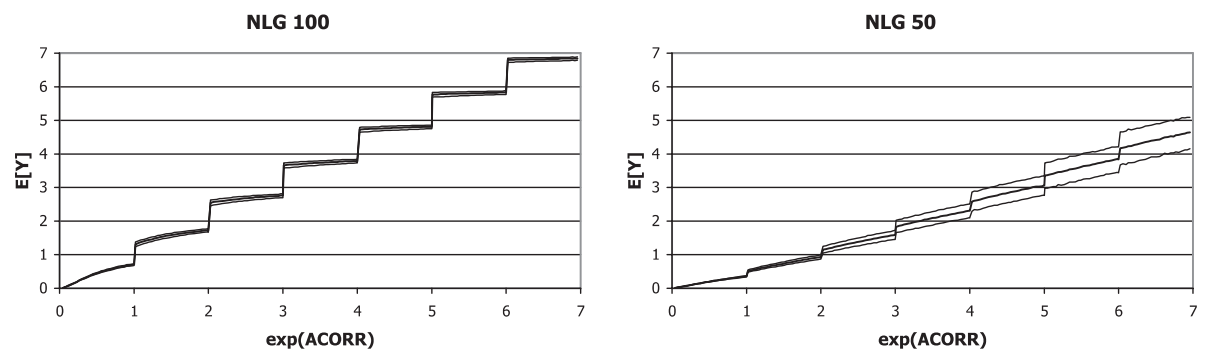

NLG 25
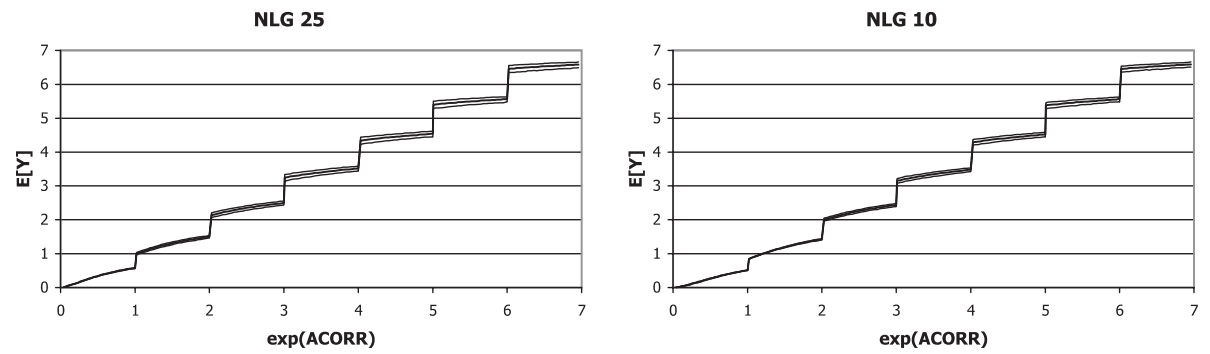

Fig. 4. $95 \%$-confidence intervals around $\mathrm{E}\left[Y_{d}\right]$ for each of the notes (lower bound equals 0 ).

as can be seen from (7) and (12). The probability that $Y_{d}$ is larger than $\exp \left(A C O R R_{d}\right)$ is always zero by definition. Hence, for $\exp \left(A C C O R_{d}\right) \leqslant 1$, the upper bound $u b_{d}$ equals 1 , for $1<\exp \left(A C O R R_{d}\right) \leqslant 2$ the upper bound $u b_{d}$ equals 2 , and so forth. To give an idea of the parameter uncertainty in the expected values, 

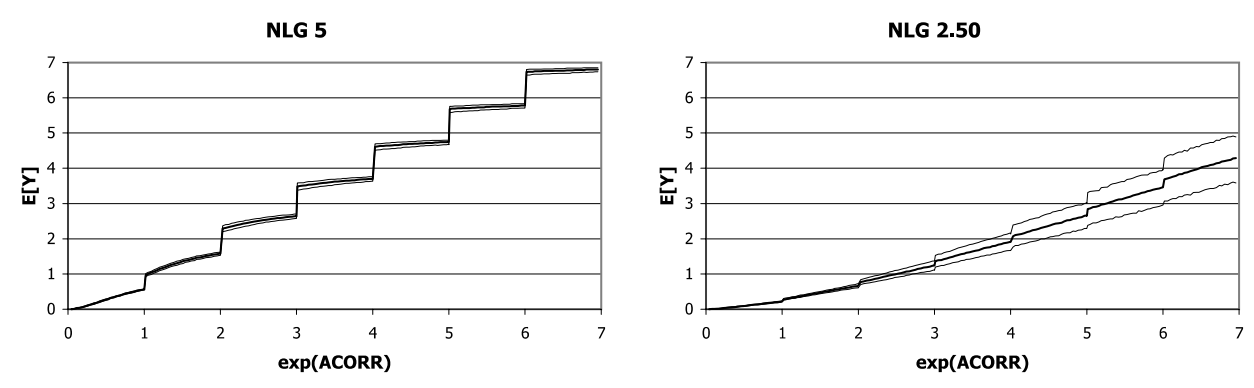

NLG 1

NLG 0.25
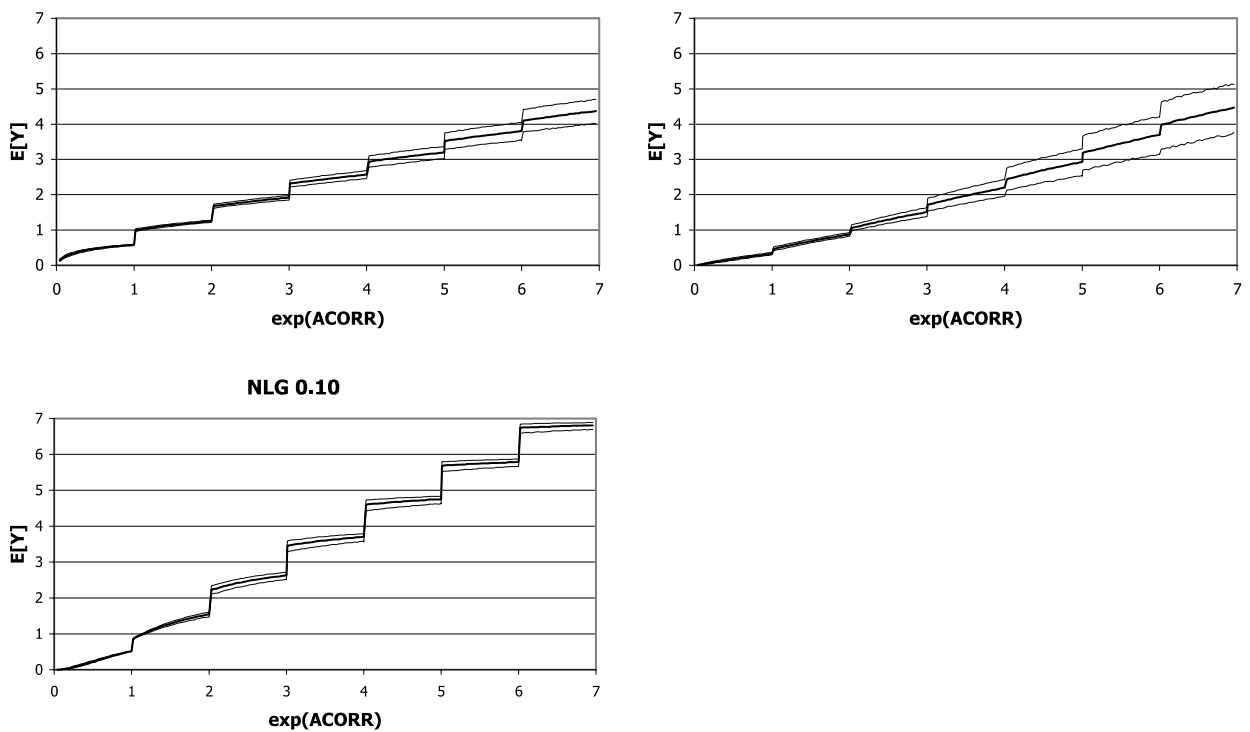

Fig. 5. 95\%-confidence intervals around $\mathrm{E}\left[Y_{d}\right]$ for each of the coins (lower bound equals 0 ).

we also plot a point-wise $95 \%$ confidence interval based on the asymptotic distribution of the parameter estimates. This confidence interval is computed using simulation. For each denomination $d$, we simulate $\beta_{d}^{(n)}$ parameter from a normal distribution with $\hat{\beta}_{d}$ as mean and the estimated heteroskedastic-consistent covariance matrix for $n=1, \ldots, N$ as covariance matrix, see also Table 5. For a given value of the $A C O R R_{d}$ we compute the choice probabilities between the upper bounds $u b_{d}$ and lower bounds 0 and construct the expected value of $Y_{d}$ as follows

$$
\mathrm{E}\left[Y_{d} ; \beta_{d}^{(n)}\right]=\sum_{z=0}^{u b_{d}} z \operatorname{Pr}\left[Y_{d}=z \mid A \operatorname{CORR}_{d}\right],
$$

where the $A C O R R_{d}$ summarizes $y_{d+1}, \ldots, y_{D}$ and we assume that there are no wallet limitations. The average value of these simulated expected values over $n$ provides the expected value of the expectation of $Y_{d}$. The $95 \%$ confidence intervals correspond to the $2.5 \%$ and $97.5 \%$ percentile of the $N$ simulated expected values. 
NLG 100 versus NLG 50

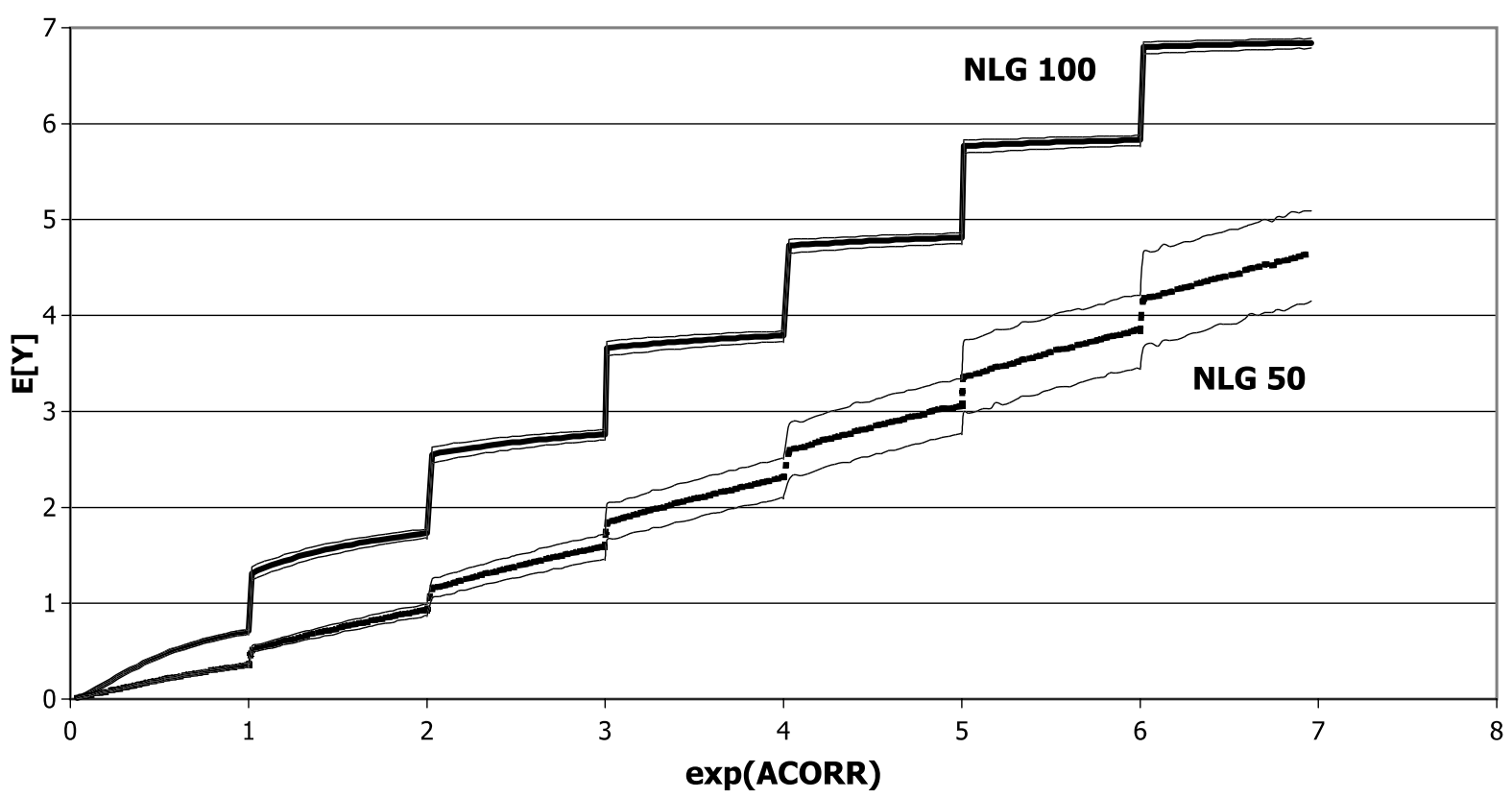

Fig. 6. $\mathrm{E}\left[Y_{50}\right]$ and $\mathrm{E}\left[Y_{100}\right]$ and their $95 \%$-confidence intervals (lower bound equals 0 ). 
Figure 2 shows the expected value for the bank notes. The expected values are increasing for all denominations. The jumps in the lines are due to the change in the upper bound as a result of the change in the $A C O R R_{d}$ variable. Similarly, Figure 3 shows the results for all coins. The same conclusion can be drawn from the positive curves for all denominations. In sum, an increase in $A C O R R_{d}$ will lead to an increase in the expected value of $Y_{d}$, for all $A C O R R_{d}$ and all notes.

The graphic representation of the estimation results in Figure 2 shows another striking result. The curve for NLG 50 lies beneath the curves of the other denominations, while the curve for NLG 100 lies above all others. This difference is significant as can be shown by taking into account the 95 percent confidence intervals of the expected value, as depicted in Figure 4 and Figure 5. To make this difference even clearer, we zoom into these two denominations in Figure 6. The conclusion can be drawn that the NLG 50 has a lower probability for being chosen as payment than NLG 100 for all values of $A C O R R$.

At this point we return to the discussion initiated in Section 4 where it was stated that the premise of indifference of individuals towards the use of denominations in payments, referred to as premise (ii) in currency research, could only be investigated by means of statistical modeling. This is because the use of denominations should be studied under equal payment circumstances for proper comparison. This is exactly what the graphic representation, as depicted in Figures 2 and 3, does. We use an example to give additional insights.

We compare denominations NLG 100 and NLG 50 by choosing a reference point at the relevant curves in Figure 2. The following holds.

\begin{tabular}{lll}
\hline & NLG 100 & NLG 50 \\
\hline to pay & NLG 200.00 & NLG 100.00 \\
$A C O R R_{d}$ & 2 & 2 \\
$\mathrm{E}\left[Y_{d}\right]$ & 2.56 & 1.82 \\
\hline
\end{tabular}

Suppose that two individuals are faced with a payment choice problem. The first has an amount of NLG 200.00 left to pay and disposes of three banknotes of NLG 100. As this individual's wallet contains enough banknotes and coins of lower denominations to pay the NLG 200.00, the lower bound for choosing to pay with NLG 100 is equal to 0 . The second individual has NLG 100.00 left to pay and needs to decide how many of his or her banknotes of NLG 50 to use. This individual also does not need to use any NLG 50 to be able to pay for the amount. The situations of the two individuals are equal, as was required for comparison. The estimated cash payment model now provides a tool to investigate the difference in use of NLG 50 and NLG 100 under equal circumstances. The results indicate that the expected value of NLG 50 will be 1.82 in this particular situation, while the expected value of 2.56 for NLG 100 is higher.

The top panel of Figure 2 shows that the expected value is higher for NLG 100 than for NLG 50 for all values of $A C O R R$. The bottom panel shows a similar difference. From this analysis using the estimation results of our cash payment model 
it can be concluded that under equal circumstances NLG 50 is less preferred (or popular) than NLG 100 in cash payments. This contradicts the premise of indifference, commonly used in currency research to derive the optimal denominational mix. A similar kind of analysis can be done for coins.

\section{Research implications}

In this paper we have put forward the first empirical analysis of individual cash payment behavior. In our opinion, research in this area is essential. Issuing and handling cash is a costly operation involving many parties. And, as we have shown in this paper, commonly applied assumptions on payment behavior do not necessarily coincide with actual payment behavior, making the issue of the optimal monetary system even more complex. By analyzing payment behavior at the individual level, it became clear that there is more to the use of cash than theoretical concepts such as efficiency or indifference imply. We intend to elaborate this type of currency research, for which we see opportunities in several directions.

We used the data at hand as a starting point for our analysis and encountered several challenges while developing our cash payment model. We needed to account for the unobserved wallets at the time of the transaction. Also, we disposed only of a limited number of explanatory variables. For improving our model estimation in further research we aim to collect data on payments and wallets at the same time. Furthermore we intend to observe characteristics of the paying individual to improve the explanatory power of our model. For this purpose, we will need to pay special attention to data collection methods that bypass privacy sensitivity. One way of collecting data would entail the design of experiments.

The prime focus of further empirical research will be the new currency that has only been circulating for a short period of time. The Euro, one of the two major currencies used worldwide, will develop in the coming years and our study will be able to compare the use of the new currency in the different European countries.

Through our study and its results, we have become intrigued by the still unresolved question of what the optimal denominational structure is. We feel that with our empirical approach of cash payment behavior we will be able to shed light on this problem by collecting data on virtual denominational ranges. Applying our modeling method we intend to draw conclusions on the preferred denominations and structures by individuals, taking into account individual payment behavior.

\section{A Appendix: Efficient payment algorithms}

In this appendix we describe the algorithm developed by Cramer (1983). Furthermore, we describe our extension of this algorithm to the situation where the individual does not have all denominations at his or her disposal. 


\section{A.1 Cramer's algorithm}

CRAMER (1983) formulates efficient payments in mathematical terms as the solution to an optimizing problem. Consider $A$ to be the amount to be paid, and $n(A)$ the combination of the different notes and coins used in the cash payment. If the different denominations in an arbitrary currency range are numbered as $d=1, \ldots, D$, then $n(A, d)$ denotes the number of tokens of denomination $d$ used for paying amount $A$. A positive $n(A, d)$ refers to use as a payment, while a negative $n(A, d)$ means that the $n$ tokens of denomination $d$ are given as change. We denote the value of denomination $d$ by $v_{d}$. Efficient payments $n(A, d)$ are then the solution to the following problem:

Minimize

$$
n(A)=\sum_{d}|n(A, d)|
$$

subject to

$$
\sum_{d} n(A, d) v_{d}=A
$$

Given the values for $v_{d}$, this problem is solved as follows. We take a range of amounts that are of interest, say NLG 0.05 to NLG 100 with intervals of NLG 0.05 . This yields a list of 2,000 amounts in total. The goal of the algorithm is to cover each amount in this list with an efficient combination of tokens. The steps of the algorithm are as follows:

1. The algorithm starts by covering all amounts in the list that can be paid by only one token. For example, in the Dutch system and with the range given above, the amounts NLG $0.05,0.10,0.25,1,2.50,5,10,25$ and 100 in our list would be covered.

2. Next, all amounts that can be paid with two tokens, either given by the consumer as payment or by the retailer as change, are computed. If in this step we find an amount that was already covered with only one token in the previous step, such as NLG $50+$ NLG 50, we do not add this pair of two tokens to the list, since two tokens is not efficient for this amount. An example amount that would be covered in this step is NLG 0.20 . The combination needed here is NLG 0.25 and NLG 0.05 as change. Another scheme with the same amount of tokens is NLG $0.10+$ NLG 0.10 . Both these example schemes are stored in this step.

3. To the pairs that were found efficient in the previous step, we add each token once, both with positive and negative sign. For a given pair, this results in $2 D$ extra potential combinations with an additional token each. Adding a token with a positive sign to a combination, which has this same token with a negative sign, would yield a combination with less tokens and is therefore ignored. Also, we have the restriction that the highest token has a positive sign.

With these combinations, we cover all resulting amounts, provided they were not already covered by less tokens.

4. We repeat step 3 until all amounts on the list are covered. 
This algorithm results in a number of efficient combinations for each possible amount in the range specified. Many amounts can be paid efficiently with more than one combination.

\section{A.2 Cramer's algorithm, modified for wallet contents}

Cramer's algorithm assumes that an individual has all denominations at his or her disposal. If we relax this assumption, that is, we include the restriction of the wallet contents of the individual, the following optimization problem, which we might call a wallet-specific algorithm results. A restricted efficient payment for a given amount $A$ and wallet $w$ is the solution to:

Minimize

$$
n(A)=\sum_{d}|n(A, d)|
$$

subject to

$$
\begin{aligned}
\sum_{d} n(A, d) v_{d} & =A \\
n(A, d) & \leqslant w_{d} \quad \forall d,
\end{aligned}
$$

where $w_{d}$ represents the number of tokens of denomination $d$ that is available to the individual in his or her wallet.

If for a certain individual $i$, his or her amount $A_{i}$ and wallet $w_{i}$ an efficient payment, as computed with the standard Cramer algorithm, can be paid (i.e. the wallet $w_{i}$ suffices to pay according to the efficient scheme), we can simply take this efficient scheme. However, if the wallet contents of the individual is not enough to pay according to the efficient scheme, we need to find the restricted efficient scheme, that is, the combination of tokens that minimizes the numbers of tokens used, given the wallet contents. In this case, the following algorithm is executed for this particular amount $A_{i}$ and wallet $w_{i}$ :

1. The algorithm starts by computing all amounts in this range that can be covered by only one token. If the wallet contents $w_{i}$ of the individual suffices, the combination is stored.

2. Next, all amounts that can be paid with two tokens, either given by the consumer or by the retailer as change, are computed. Again, only if the wallet contents of the individual suffices, the combination is stored.

3. To the pairs that were found efficient in the previous step, we add each token once, both with positive and negative sign. With these combinations, we fill the list with the resulting amounts, provided they are not already covered by less tokens, and provided the combination fits in the individual's wallet $w_{i}$.

4. We repeat step 3 until the amount $A_{i}$ is covered. Note that it is not enough to store only the combination which pays the given amount $A_{i}$ exactly. We need to store each combination that fits in the individual's wallet, since we use these as the starting point for the schemes in the next step. 
This algorithm is executed for each transaction for which the wallet does not suffice to pay according to the standard efficient scheme.

\section{References}

Caianiello, E., G. Scarpetta and G. Simoncelli (1982), A systemic study of monetary systems, International Journal of General Systems 8, 81-92.

Cramer, J. (1983), Currency by denomination, Economics Letters 12, 299-303.

Hove, L.V. (2001), Optimal denominations for coins and bank notes: in defense of the principle of least effort. Journal of Money, Credit and Banking 33, 1015-1021.

Hove, L.V. and B. Heyndels (1996), On the optimal spacing of currency denominations, European Journal of Operational Research 90, 547-552.

Sumner, S. (1993), Privatizing the mint, Journal of Money, Credit and Banking 25, 13-29.

Telser, L.G. (1995), Optimal denominations for coins and currency, Economics Letters 49, 425-427.

White, H. (1980), A heteroskedasticity-consistent covariance matrix estimator and a direct test for heteroskedasticity, Econometrica 48, 817-828.

Wynne, M.A. (1997), More on optimal denominations for coins and currency, Economics Letters 55, 221-225.

Received: December 2002. Revised: April 2003. 